ARTICLE

\title{
Circularly polarised luminescence laser scanning confocal microscopy to study live cell chiral molecular interactions
}

\author{
Patrycja Stachelek (10 ${ }^{1,2}$, Lewis MacKenzie (iD ${ }^{1,2}$, David Parker (iD ${ }^{1} \&$ Robert Pal $^{1 凶}$
}

The molecular machinery of life is founded on chiral building blocks, but no experimental technique is currently available to distinguish or monitor chiral systems in live cell bioimaging studies. Luminescent chiral molecules encode a unique optical fingerprint within emitted circularly polarized light (CPL) carrying information about the molecular environment, conformation, and binding state. Here, we present a CPL Laser Scanning Confocal Microscope (CPL-LSCM) capable of simultaneous chiroptical contrast based live-cell imaging of endogenous and engineered CPL-active cellular probes. Further, we demonstrate that CPL-active probes can be activated using two-photon excitation, with complete CPL spectrum recovery. The combination of these two milestone results empowers the multidisciplinary imaging community, allowing the study of chiral interactions on a sub-cellular level in a new (chiral) light.

\footnotetext{
${ }^{1}$ Department of Chemistry, Durham University, South Road, Durham DH1 3LE, UK. ${ }^{2}$ These authors contributed equally: Patrycja Stachelek, Lewis MacKenzie

凶email: robert.pal@durham.ac.uk
} 
C hirality is intrinsic to the fundamentals of life, with chiral molecules playing pivotal roles in natural cell homoeostasis, chemical signalling, enzymatic reactions, gene expression and reproduction ${ }^{1}$. Selective chiral recognition of vital bio-molecules and bioprocesses by specifically designed probes in biological environments, like live cells, is a topic of great interest from both biochemical and analytical application points of view. Tracking chiral molecules within live cells can be of great importance to understanding interactions between cell organelles and drugs or chiral probes. It has been shown that chirality can have a profound effect on drug activity and on localisation in cells amongst others. Stereoselectivity, particularly enantioselectivity is of crucial importance when designing either drugs and/ or molecular probes to interact with proteins ${ }^{2,3}$. Despite life being founded on chiral building blocks, currently, there are no direct techniques capable of distinguishing and tracking the behaviour of enantiomers in chiral molecular systems-e.g. luminescent probes or proteins-and their individual interactions within living cells.

Circular polarisation luminescence (CPL) spectroscopy and circular dichroism (CD) spectroscopy are optical spectroscopic tools for studying chiral molecules and their interactions, but to date, these have not met the complex biocompatible requirements needed to study and track chiral interactions within cells. CD spectroscopy quantifies how chiral molecules absorb left and right circularly polarised light differentially. CD microscopes have been developed and applied to applications such as the microscopy of inorganic chiral substrates and structures, but CD microscopes face several limitations, including artefacts associated with the linearly polarised light components and optical distortion ${ }^{4-7}$. Fundamentally, CD techniques are limited by the optical absorbance of the sample, rendering $\mathrm{CD}$ too insensitive and of little use for live-cell imaging.

On the other hand, CPL spectroscopy enables the study of chiral interactions of luminescent molecular systems. Efficient CPL probe complexes, foremost lanthanide coordination complexes, can be used for cellular imaging because of their demonstrated biocompatibility and unique photophysical and CPL properties ${ }^{8}$. The chirality of such complexes and their binding motifs enables them to be exploited as sub-cellular chiral probes 9 . However, CPL spectroscopy and measurement technology has lagged behind the development of CPL-active probes, hindering the translation to sub-cellular CPL microscopy. Since the inception of CPL spectrometers roughly 50 years ago photoelastic modulators (PEMs) have been incorporated for circular polarisation analysis, in combination with scanning monochromators for wavelength specificity. Such setups have resulted in bulky, expensive instruments with slow spectral data acquisition rates $(\sim 1 \mathrm{~h} \text { for a typical CPL spectrum })^{10,11}$. Consequently, the few CPL microscopes which have been developed incorporate PEMs, and they, therefore, suffer from inherently slow-acquisition rates which are incompatible with imaging CPL within living cells due to: (a) high-energy excitation (e.g. UV/ blue) induced phototoxicity and (b) correspondingly poor spatial resolution owing to increased acquisition times. Consequently, circularly polarised emission microscopy studies have been limited to investigations of inert, bulk samples at the millimetre scale, e.g. large inorganic crystals ${ }^{12}$, or imaging maps of chiral amino acids distributed within agarose gel ${ }^{13}$. This situation is in stark contrast to the many advances in optical microscopy over recent decades, which have enabled diffraction-limited and superresolved cellular imaging ${ }^{14}$.

To advance CPL microscopy, we have previously built a proof-ofconcept chiroptical contrast time-resolved epifluorescence microscope and demonstrated enantioselective differential chiral contrast (EDCC) imaging, examining the $\Lambda$ - and $\Delta$-enantiomers of a bright
CPL-active europium complex (Eu:L1, Eu[1,4,7- tris(\{4-[2-(4methoxy-2- methylphenyl)ethynyl]-6-[carboxy(phenyl)phosphoryl] pyridin-2-yl $\}$ methyl)-1,4,7- triazacyclononane]) (structure depicted on Fig. 3) absorbed onto an optical brightener-free paper test substrate ${ }^{9}$. This study showed that the enantiomers could be spatially discriminated by their CPL emission profile. This epifluorescence CPL microscope was considerably faster than PEM-CPL microscopes ( $2 \mathrm{~min}$ vs. $40 \mathrm{~min}$ total image acquisition), but still required sequential manual selection of CPL detection channels, which is impractical for sub-cellular imaging where high spatio-temporal resolution is necessary to track chiral interactions without the introduction of image artefacts. A microscope capable of simultaneously measuring left and right-handed CPL (ideally with confocal sectioning and truly diffraction-limited resolution) represents a stepchange in technological capability, opening up new opportunities to study chiral molecular interactions.

We recently introduced next-generation CPL spectrometer technology which circumvents the aforementioned hindrances of 20th century CPL spectroscopy technology, using a patented optical layout and rapid charge-coupled device (CCD) spectrometers ${ }^{15}$.

Here, we demonstrate the extension of this rapid CPL measurement technology to CPL laser scanning confocal microscopy (CPL-LSCM). This approach allows the simultaneous acquisition of left and right-handed CPL images for enantioselective differential contrast microscopy, with a diffractionlimited spatial resolution (i.e. $126 \mathrm{~nm}$ lateral, $396 \mathrm{~nm}$ axial resolution using $\times 63 \quad 1.4$ NA (numerical aperture) objective) across a typical field of view (FOV) of $100 \times 100 \mu \mathrm{m}$ in $9 \mathrm{~s}$, when employing $355 \mathrm{~nm}$ single-photon activation with an $\mathrm{Nd}$ :YAG (3rd harmonic) laser. The CPL-LSCM could be a radical new tool in bio-imaging enabling the study of fundamental chiral interactions within live cells by harnessing the unique and uncharted power of CPL. In addition to cellular applications, CPL-LSCM could be used in studies of thin and thick film embedded CPL emitters, for applications such as physically unclonable multi-layered CPL-active security inks ${ }^{16,17}$, and next-generation organic light-emitting diodes ${ }^{18}$.

\section{Results}

Throughout this work when discussing europium CPL, we selected the magnetic dipole $(\mathrm{MD})$ allowed $\Delta J=1$ transition $(\lambda \sim 595 \mathrm{~nm})$ because it exhibits both high overall emission intensity and a high $g_{\text {em }}$ value (i.e. an overall high CPL brightness) for the employed Eu:complexes. In this $\mathrm{MD}$ allowed transition manifold around $595 \mathrm{~nm}$, europium complexes with transitions of the same sign of CPL emission have been identified. Therefore, using optical filters, the whole $\Delta J=1$ manifold can be selected maximising light collection efficiency; it represents the best emission window to examine for Eu complexes in CPL-LSCM.

Multi-photon (MP) CPL spectroscopy. CPL spectroscopy is a well-established discipline, with CPL emission maximised for emission transitions which are MD allowed and electric dipole (ED) forbidden. CPL emission is most commonly quantified in terms of the emission dissymmetry factor, $g_{\text {em }}$, calculated by

$$
g_{\mathrm{em}}=\frac{2\left(I_{\mathrm{L}-\mathrm{CPL}}-I_{\mathrm{R}-\mathrm{CPL}}\right)}{\left(I_{\mathrm{L}-\mathrm{CPL}}+I_{\mathrm{R}-\mathrm{CPL}}\right)} .
$$

Where $I_{(\mathrm{L}-\mathrm{CPL})}$ and $I_{(\mathrm{R}-\mathrm{CPL})}$ are the intensities of (left-handed) L-CPL and (right-handed) R-CPL emission; $g_{\mathrm{em}}=2$ indicates $100 \% \mathrm{~L}-\mathrm{CPL}$ emission, $g_{\mathrm{em}}=-2$ indicates $100 \% \mathrm{R}-\mathrm{CPL}$ emission, and $g_{\mathrm{em}}=0$ indicates net-zero circular polarisation. The strongest CPL signals to date have been generated by lanthanide coordination complexes, specifically europium complexes, exhibiting $g_{\mathrm{em}}=|1.38|$ for single complexes and $g_{\mathrm{em}}=|1.45|$ for 
chiral supramolecular polymers ${ }^{19,20}$. Recently, the more comprehensive metric of circularly polarised brightness $(\mathrm{CPB})$ has been introduced ${ }^{16,21}$. CPB serves as a metric for the total number of circularly polarised photons emitted and combines the molar absorption coefficient $\left(\xi_{\mathrm{abs}}\right)$, quantum yield $\left(\phi_{\mathrm{em}}\right)$, and $g_{\text {em }}$. CPB is calculated as

$$
\mathrm{CPB}=\xi_{\mathrm{abs}} \phi_{\mathrm{em}}\left(g_{\mathrm{em}} / 2\right)
$$

It has units of $\mathrm{cm}^{-1} \mathrm{M}^{-1}$. A wide variety of CPL emitting molecular systems can produce useable $\mathrm{CPB}$ values (loosely defined here as $\sim 50 \mathrm{~cm}^{-1} \mathrm{M}^{-1}$ ), including BODIPYs, helicenes, excimers, cyclophanes, and d-metal complexes ${ }^{21}$. However, for the purposes of CPL-LSCM, chiral lanthanide complexes are pre-eminent owing to their excellent photophysical properties: large Stokes' shifts, narrow, line-like emission spectra, tunable emission and excitation properties, intricate CPL emission, with the potential for exceptional $g_{\text {em }}$ and CPB values (e.g. CPB values of $>1000$ and $>3000 \mathrm{~cm}^{-1} \mathrm{M}^{-1}$ with europium complexes) $)^{5}$. However, CPL spectroscopy to date has been concerned with single-photon excitation, which is sub-optimal for cellular imaging studies due to the associated potential photo-damage and shallow tissue penetration of near-UV excitation.

Multiphoton (MP) spectroscopy and microscopy techniques reduce cellular phototoxicity by harnessing a tightly focused lowenergy near infra-red (NIR) laser pulse (typically femtoseconds) to stimulate emission from fluorescent/luminescent molecules that would otherwise require absorption of a single high-energy UV photon $^{22}$. The tightly defined spatial constraints of MP microscopy offers both improved axial (z-axis) resolution and sub-cellular sectioning capability for imaging and photodynamic therapy applications ${ }^{23}$. However, the field of MP-CPL has been largely unexplored. We are aware of just one previous report of an MP upconversion-CPL spectrum in the literature of chiral perovskite nanocrystals ${ }^{24}$.

Two-photon excitation (2PE) CPL spectroscopic studies of a europium (III) complex were undertaken by coupling a tunable femtosecond pulsed laser (680-1300 nm, Coherent Discovery TPC, $100 \mathrm{fs}, 80 \mathrm{MHz}$ ) to two pre-existing CPL spectrometers. (1) a conventional $\mathrm{PEM}^{25} \mathrm{CPL}$ spectrometer, and (2) a next-generation solid-state CCD (SS-CCD) CPL spectrometer ${ }^{15}$. Either approach yielded the same CPL spectrum, measuring a model CPL complex, modified Eu:BPEPC (Eu[(4-methoxyphenyl)alkynyl-bpepc $\left.]_{3}\right\} \mathrm{Cl}_{3}$ Fig. 1$)^{26}$, but as per previous publications, the SS-CCD-CPL spectrometer acquired the CPL spectrum 800 times faster than the PEM-CPL spectrometer (under $10 \mathrm{~s}$ for a 1000 accumulation spectrum). For the purposes of this work, we will report $2 \mathrm{PE}-\mathrm{CPL}$ spectra from the PEM CPL spectrometer because this form of CPL spectrometry represents the current benchmark instrumentation of the CPL spectroscopy community.

For efficient two-photon excitation, a material must have a high two-photon absorption (TPA, 2PA) cross-section $\left(\sigma_{2}\right)(\mathrm{GM})$ with a favourable emission quantum yield. Therefore, we extend $\mathrm{CPB}$ to two-photon-activation $\mathrm{CPB}\left(2 \mathrm{PE}-\mathrm{CPB}\right.$ or $\left.\mathrm{CPB}_{2 \mathrm{PE}}\right)$ as

$$
2 \mathrm{PE}-\mathrm{CPB}=\sigma^{2} \times \phi_{e m} \times \frac{g_{\mathrm{em}}}{2}
$$

Certain lanthanide complexes (especially those of Eu(III) and $\mathrm{Tb}(\mathrm{III})$ ) have adequate two-photon cross-sections ${ }^{27}$, leading to good $2 \mathrm{PE}-\mathrm{CPB}$ values, paving the way towards two-photon activated live-cell CPL-LSCM. We determined the crosssections $\left(\sigma^{2}\right)$, of the standard materials (modified Eu:BPEPC and Eu:L1) discussed herein ${ }^{26,28}$, according to established procedures (see SI ${ }^{27,29}$. It is important to note that due to the nonlinear effect of $2 \mathrm{PE}$ (non-degenerate two-photon absorption) the 2PE wavelength is often lower and extremely rarely double of the $1 \mathrm{PE}$ absorption maximum. Due to the quadratic relationship between the intensity of the $2 \mathrm{PE}$ excitation and the triggering of a fluorescent event the shape of $2 \mathrm{PE}$ excitation is inherently always far narrower, sharper too than that of the 1PE excitation ${ }^{23,29}$.

We verified that the MP excitation process was a two-photon event by recording an excitation power dependence; confirmed by the resulting line having a slope of $\sim 2$ on a logarithmic scale (see Fig. 1A). We measured the MP cross-section of the recently developed CPL-standard Eu(III)-complex, (modified Eu:BPEPC) via these methods (Fig. 1). Its $2 \mathrm{PE}$ cross-section was determined to be $\sigma^{2}=142 \pm 3$ and $147 \pm 3 \mathrm{GM}\left(1 \mathrm{GM}=10^{-50} \mathrm{~cm}^{4}\right.$ s photon $\left.{ }^{-1}\right)$ for the $\Lambda$ - and $\Delta$-enantiomers in methanol (MeOH), respectively. This result is particularly notable because MP cross-sections have not previously been determined for enantiomers of CPL active materials. Eu:L1, is a complex which has shown enantioselective localisation in live-cell experiments ${ }^{27,30}$. The MP cross-section of Eu:L1 in $\mathrm{MeOH}$ was determined to be $\sigma^{2}=51 \pm 3 \mathrm{GM}$ for both the $\Lambda$ - and $\Delta$-enantiomers (see SI Fig. 1).

Enantioselective CPL-LSCM microscopy imaging. Details of the CPL-LSCM set-up are shown in Fig. 2. In brief, light from the sample plane of the LSCM exits the microscope via an output port and into an external CPL analysis module, which is adapted from the rapid CPL spectrometer that we recently reported ${ }^{15}$. First, the waveband of interest is selected by a switchable bandpass filter. Then, an achromatic wave plate converts left and right circularly polarised light into orthogonal linearly polarised states. The light is then split into two analysis pathways by a 50:50 beam-splitter cube. The two linear polarised light states generated (horizontal and vertical polarisation) corresponding to left or right CPL are selected by a carefully aligned linear polarizer, housed in a high-precision computer-controlled rotation mount ${ }^{15}$. The emission intensity of each pixel is quantified in a conventional LSCM scanned manner by a dedicated high sensitivity avalanche photodiode pair. Whilst each detection arm can operate independently, both components are matched in alignment and specification to enable rapid and simultaneous acquisition of left and right CPL images. Full technical details of the CPL-LSCM system and the applied image processing methodology are provided in the Supporting information.

EDCC imaging was realised by subtraction of the simultaneously recorded left-handed CPL image from the right-handed CPL (and vice versa) using ImageJ software (v1.49) ${ }^{31}$.

To demonstrate the CPL-LSCM on well-controlled targets, we first re-created the paper test-target [Test Target 1] which had been used for the proof-of-concept CPL microscopy studies reported in $2016^{9}$. This was achieved by simply depositing $15 \mu \mathrm{L}$ of enantiopure solutions of Eu:L1 $\left(10^{-4} \mathrm{M}\right)$ in $\mathrm{MeOH}$ onto the commercially available optical brightener-free paper substrate from Canson ${ }^{\circledast}$, allowing the sample to dry at room temperature. This procedure replicated the results obtained with the epifluorescence microscope and further demonstrated the excellent performance of the CPL-LSCM. The experiment revealed differences arising from the texture of the brightener-free paper. Under the applied $\times 40(0.7 \mathrm{NA}$, air) magnification, it was found that the paper has a 'rough' and 'smooth' side, which was not observed in prior studies with the epifluorescence CPL microscope (see SI Figs. 4-6 and 8) ${ }^{9}$. To remove the variables introduced by the paper substrate, another test-target was developed [Test Target 2] where enantiopure solutions of Eu:L1 $\left(5 \times 10^{-5} \mathrm{M}\right)$ were deposited into a uniform polymer matrix (Polyvinylpyrrolidone (PVP-40), Sigma-Aldrich) $)^{9,28}$ by spincoating on glass substrates $(170 \mu \mathrm{m}$ thick standard microscope coverslips). Distribution of the complexes within a polymer matrix is of particular interest for ultra-secure anti-counterfeiting 
A
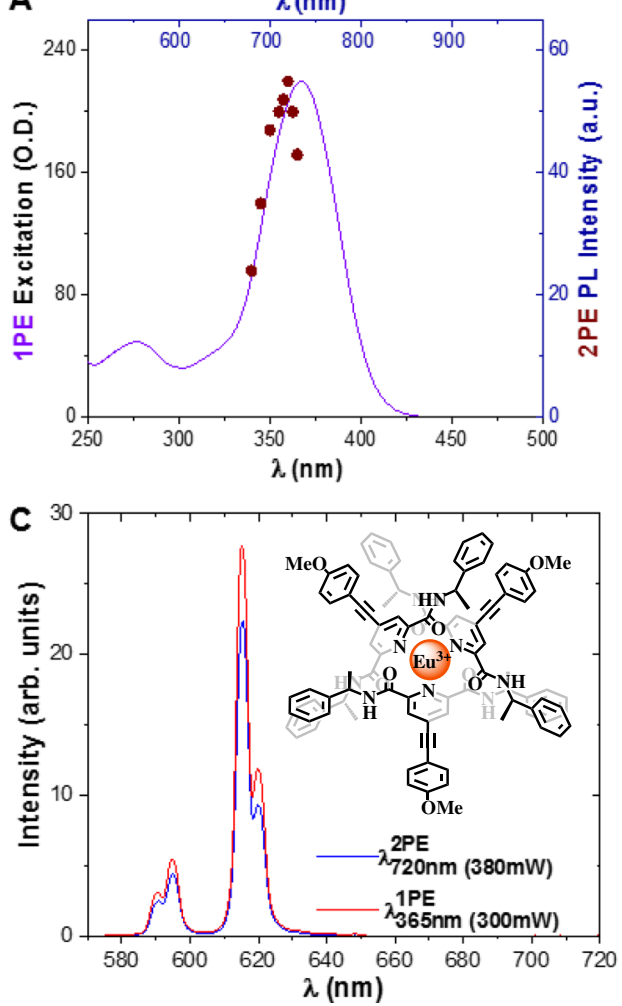

B
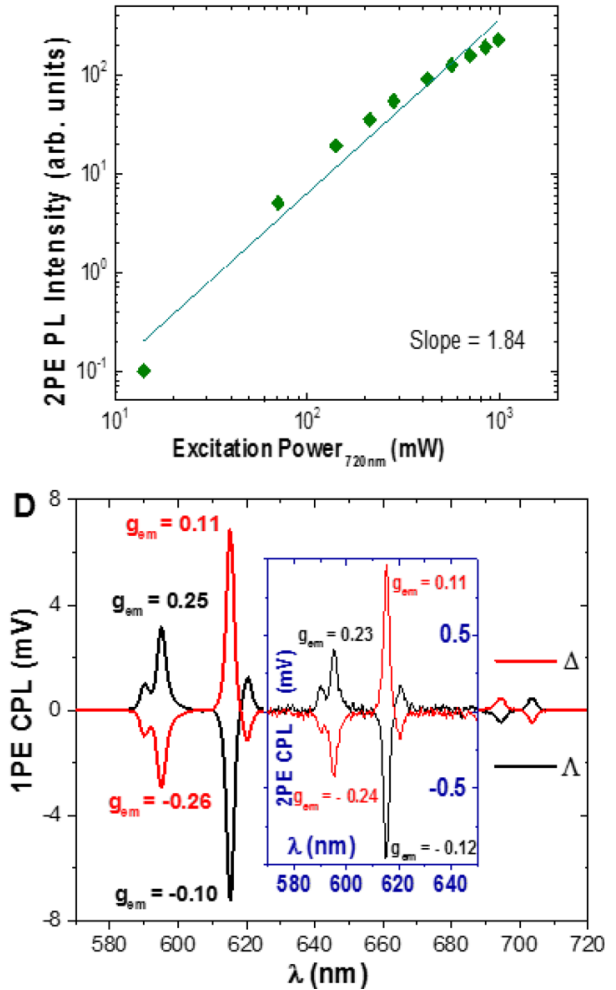

Fig. 1 Key photophysical parameters and spectra of $\boldsymbol{\Lambda}$ - and $\boldsymbol{\Delta}$-modified Eu:BPEPC in MeOH. A One photon excitation (solid purple line) and two-photon excitation (maroon dots) spectra $\left(\lambda_{\mathrm{em}}=615 \mathrm{~nm}\right.$ ) of modified Eu:BPEPC. B Excitation power dependency (green diamonds) of the 2PE induced photo luminescence (PL) intensity, slope $1.84 \pm 0.1, \sigma^{2}=144.5 \pm 3 \mathrm{GM}\left(10^{-50} \mathrm{~cm} 4 \mathrm{~s} /\right.$ photon $)$. C One $\left(\lambda_{\text {ex }}=365 \mathrm{~nm}\right.$, solid red line $)$ and two-photon $\left(\lambda_{\mathrm{ex}}=720 \mathrm{~nm}\right.$, solid blue line) induced emission spectrum of the depicted (insert) $\Lambda$ - and $\Delta$ Eu:BPEPC. D One photon and (insert) two-photon CPL spectra of $\Lambda$ - and $\Delta$-modified Eu:BPEPC (solid black and red line respectively). Spectra recorded in $\mathrm{MeOH}$.

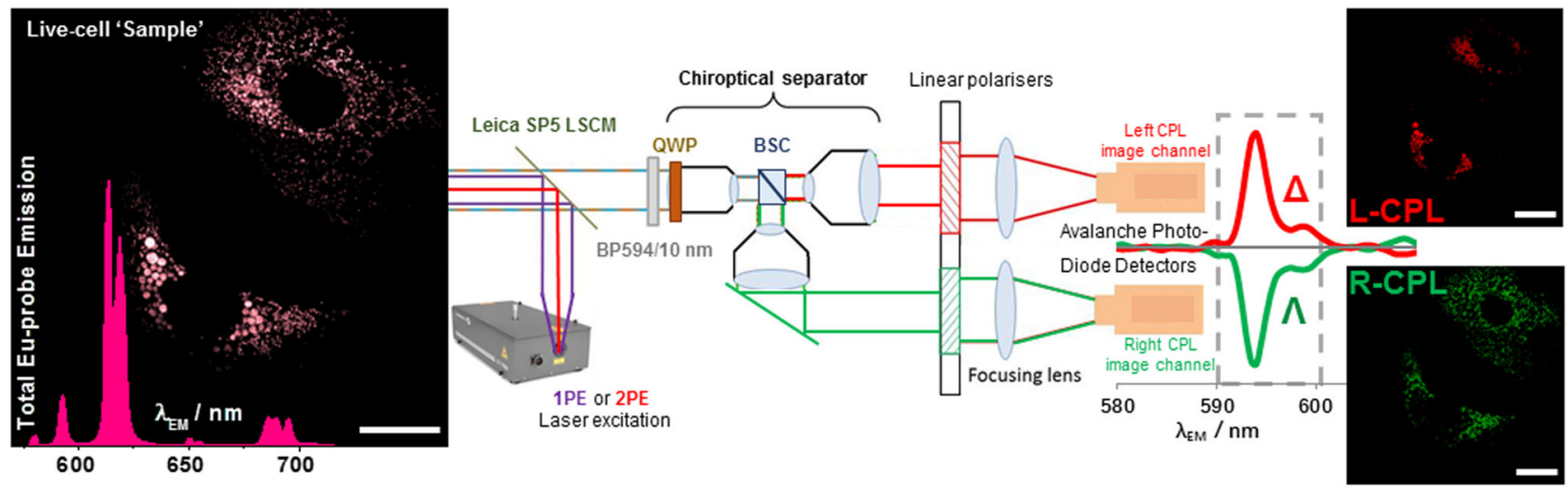

Fig. 2 Simplified depiction of the CPL-LSCM developed for enantioselective differential chiral contrast (EDCC) imaging. The external CPL-LCSM module is attached to a commercial LSCM via a dedicated external (X1) port to facilitate simultaneous parallel diffraction-limited enantioselective imaging of CPL-active probes within live cells. Scale bars $=20 \mu \mathrm{m}$.

CPL-active security ink applications (e.g. for application within plastic banknotes) and solution-processed CPL-OLEDs.

To demonstrate that Eu:L1 retains its distinctive emission properties within the PVP40 polymer, we measured CPL emission, 2PE-CPL emission, and 2PE-cross-section measurements of Eu:L1 in anhydrous N-methyl-2-pyrrolidone (NMP) (see Fig. 3). NMP was selected because NMP is structurally almost identical to the monomer unit of PVP polymers $(\mathrm{N}$ Vinylpyrrolidone). This is crucial because it has recently been reported that CPL signal intensity and even CPL sign may vary as a function of solvent or medium polarity ${ }^{32}$. We found that the CPL signal intensity $\left(\left|g_{\mathrm{em}}\right|\right.$ and $\left.\mathrm{CPB}\right)$ and sign were unaltered and the 2PE cross-section of Eu:L1 was even slightly enhanced in NMP $\left(\sigma^{2}=73 \pm 3 \mathrm{GM}\right.$ for $\Lambda$ - and $\Delta$-enantiomer $)$ compared to $\mathrm{MeOH}\left(\sigma^{2}=51 \pm 3 \mathrm{GM}\right.$ for $\Lambda$ - and $\Delta$-enantiomer), validating our choice of host polymer for spin-coated test targets.

However, the non-uniform edges of the glass substrates caused light helicity inversion at the edges of the glass substrates owing to unwanted internal reflection, making them less than ideal for side-by-side demonstration of enantioselective CPL-LSCM. This behaviour is ascribed to dramatically reduced $g_{\text {em }}$ values observed from Test Target 2 as a result of reflected light-induced CPL helicity inversion (see SI Figs. 7 and 8). 
A

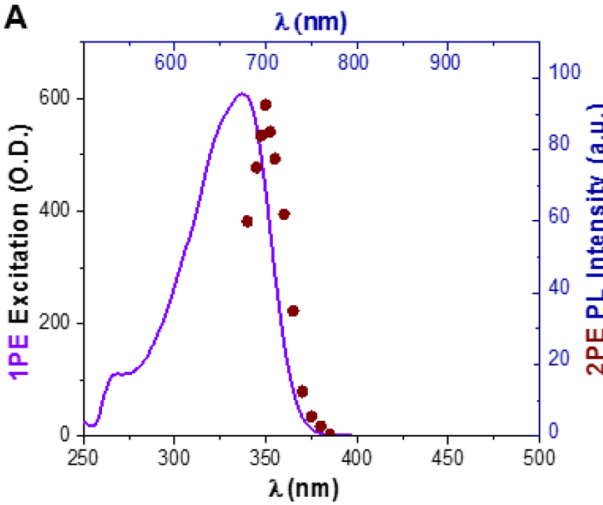

C

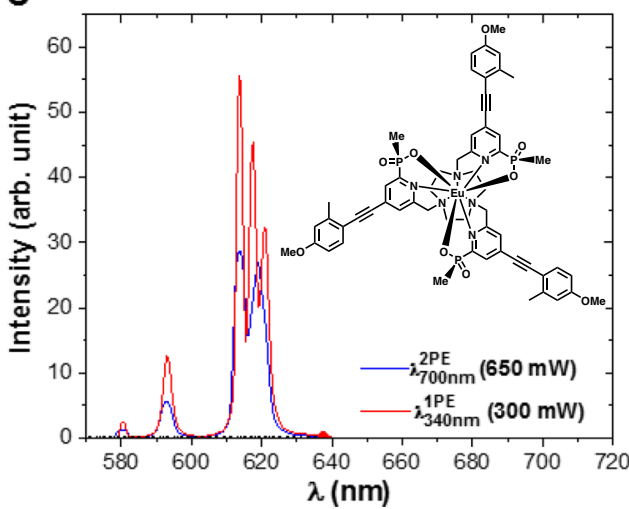

B

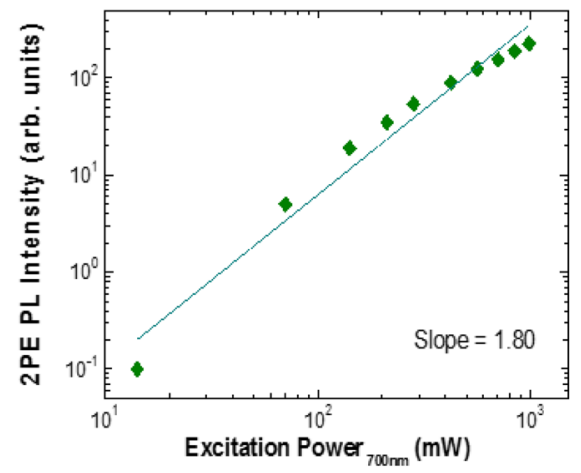

D

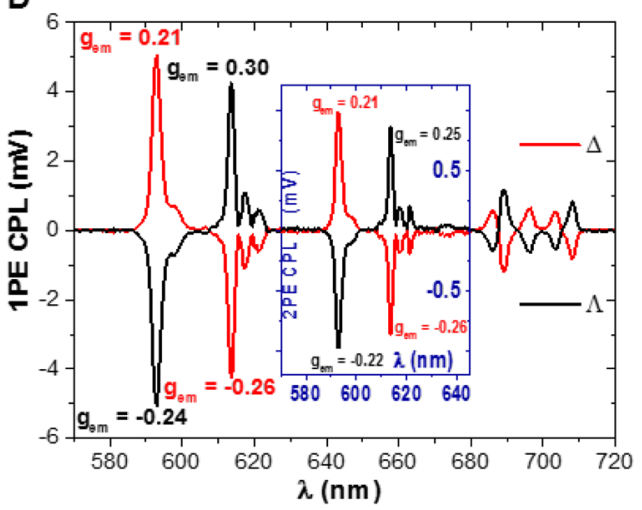

Fig. 3 Key photophysical parameters and spectra of $\boldsymbol{\Lambda}$ - and $\boldsymbol{\Delta}$ Eu:L1 in NMP. A One photon excitation $\left(\lambda_{\mathrm{em}}=615 \mathrm{~nm}\right)(\mathrm{solid}$ purple line) and two-photon excitation (maroon dots) spectra $\left(\lambda_{\mathrm{em}}=615 \mathrm{~nm}\right.$ ) of Eu:L1. B Excitation power dependency (green diamonds) of the 2PE induced photoluminescence (PL) intensity, slope $1.80 \pm 0.1, \sigma^{2}=73 \pm 3 \mathrm{GM}\left(10^{-50} \mathrm{~cm}^{4} \mathrm{~s} /\right.$ photon). C One $\left(\lambda_{\mathrm{ex}}=340 \mathrm{~nm}\right.$, solid red line) and two-photon induced $\left(\lambda_{\mathrm{ex}}=700 \mathrm{~nm}\right.$, solid blue line) emission spectrum of the depicted (insert) $\Lambda$ - and $\Delta$ Eu:L1. D One photon (main figure) and (insert) two-photon CPL spectra of $\Lambda$ - and $\Delta$ Eu:L1 (solid black and red line, respectively). Spectra recorded in NMP.
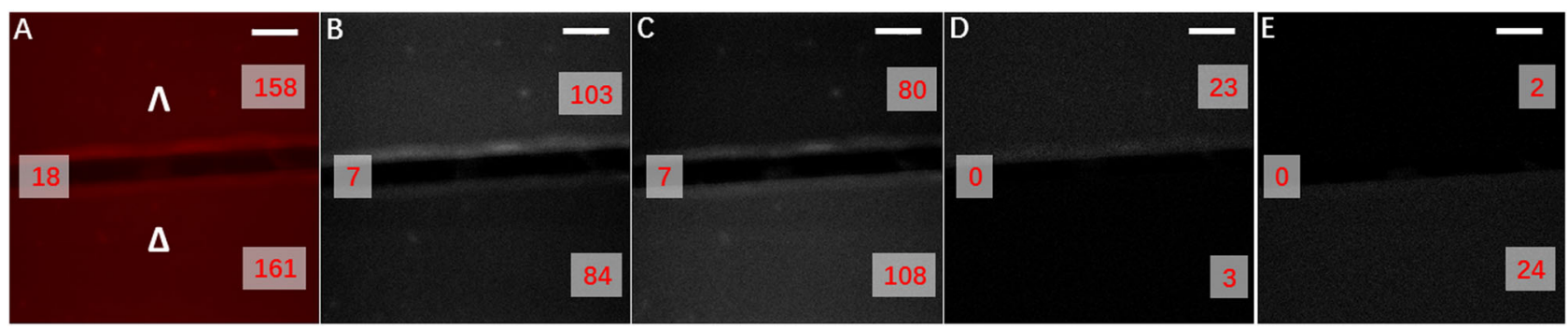

Fig. 4 Enantioselective differential chiral contrast (EDCC) CPL-LSCM of $\boldsymbol{\Lambda}$ - and $\boldsymbol{\Delta}$-Eu:L1 on a glass substrate. A Total europium emission $\left(\lambda_{\mathrm{ex}}=355 \mathrm{~nm}\right.$, $20 \mathrm{~mW}, \lambda_{\mathrm{em}}=589$ to $720 \mathrm{~nm}$ ). B, C Left and Right Handed CPL channel respectively ( $\lambda_{\mathrm{em}}=589$ to $599 \mathrm{~nm}$ ). D Left-handed EDCC image (left CPL-right $\mathrm{CPL}$ ). E Right-handed EDCC image (right CPL-left CPL). The objective used: $\times 400.7 \mathrm{NA}$ air, $210 \times 210 \mu \mathrm{m}$ FOV, 100 avg, $1.5 \mu \mathrm{m}$ axial section. Scale bars $=30 \mu \mathrm{m}$, numbers in red are avg. Eight-bit pixel intensity values for each image region.

A third test target was therefore developed with improved properties [Test Target 3]. This was achieved by heating a glass microscope slide to $35^{\circ} \mathrm{C}$ on a hot plate, and drop-casting $100 \mu \mathrm{L}$ of solutions of the $\Lambda$ - and $\Delta$-enantiomers $\left(10^{-4} \mathrm{M}\right)$ 1:1 v/v Eu:L1:PVP40 solutions in MeOH side-by-side onto the slide using a multi-channel pipette. The slide was dried on the hot plate for $1 \mathrm{~min}$, removed and allowed to dry at room temperature for $5 \mathrm{~min}^{9}$.

Test Target 3 was successfully used during validation of the CPL-LSCM setup and provided superior quality diffractionlimited images (see Fig. 4 and SI Fig. 7). Simultaneous enantioselective imaging was thereby achieved in the same FOV using bright CPB CPL emitters in a validated and controlled test target of enantiopure lanthanide complexes as homogenous thin film deposits (from a $210 \times 210 \times 2 \mu \mathrm{m}$ FOV). Additionally, we measured the total CPL spectrum of the Test Target 3 using the PEM-CPL spectrometer (see SI Figs. 2 and 3), showing excellent correspondence with respect to both $\mathrm{CPL}$ sign and $\mathrm{CPB}$.

Enantioselective CPL-LSCM of live cells. To demonstrate the live-cell imaging capabilities of the newly constructed CPLLSCM, NIH 3T3 (mouse skin fibroblast, ATCC-CRL-1658) cells were dosed with $30 \mu \mathrm{M}$ of enantiopure $\Lambda$ - and $\Delta$-Eu:L1 complex for $14 \mathrm{~h}$ as per Frawley et al. ${ }^{28}$. The images in Fig. 5 demonstrate that CPL-LSCM can simultaneously collect images and differentiate between the left- and right-handed CPL emission arising from the cells. The enantiomers of $\Lambda$ - and $\Delta$-Eu:L1 appeared to localise to different organelles with well-resolved brightness differences observable within NIH 3T3 cells. 


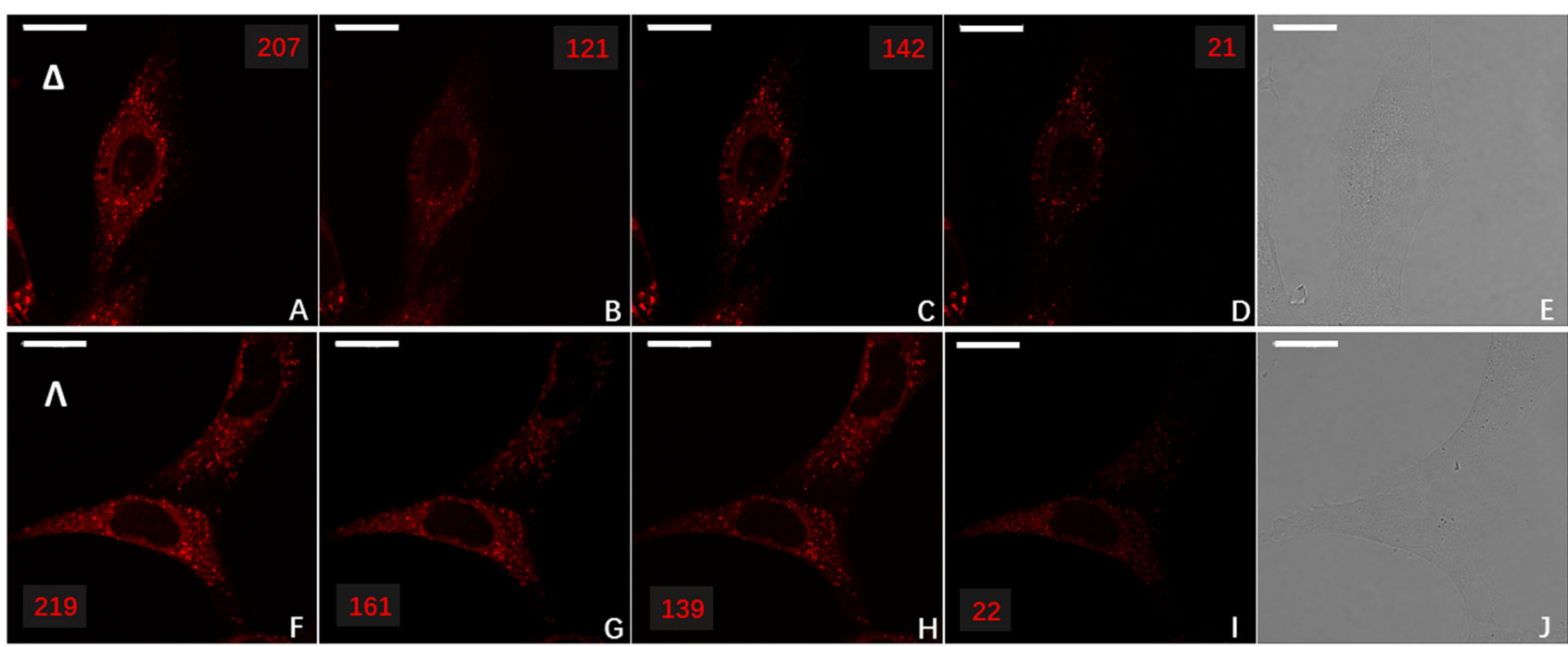

Fig. 5 Enantioselective localisation of enantiopure europium complex, Eu:L1 to the lysosome or mitochondria in live NIH $3 T 3$ cells. Enantioselective differential chiral contrast (EDCC) CPL-LSCM of Eu:L1 (30 $\mu \mathrm{M}, 14 \mathrm{~h}$ loading, $\times 631.4$ NA oil objective, $96 \times 96 \mu \mathrm{m}$ FOV, $100 \mathrm{avg}$., $790 \mathrm{~nm}$ axial section) in live mouse skin fibroblast ( $\mathrm{NIH} 3$ 3T3) cells, showing enantioselective localisation to the lysosome ( $\Delta$-Eu:L1) and mitochondria ( $\Lambda$-Eu:L1). Top row $\Delta$-Eu:L1. A Total Europium emission ( $\left.\lambda_{\mathrm{ex}}=355 \mathrm{~nm}, 20 \mathrm{~mW}, \lambda_{\mathrm{em}}=589-720 \mathrm{~nm}\right)$. B, C Left and Right CPL channel $\left(\lambda_{\mathrm{ex}}=355 \mathrm{~nm}, \lambda_{\mathrm{em}}=589-599 \mathrm{~nm}\right)$, respectively. D Left-handed EDCC image (left CPL-right CPL) highlighting enantioselective predominantly lysosomal localisation. E Transmission image. Bottom row $\Lambda$ Eu:L1. F, G, H, J as per (A, B, C, E). I Left-handed EDCC image (left CPL-right CPL) highlighting enantioselective predominantly mitochondrial localisation. Scale bars $=20 \mu \mathrm{m}$, numbers in red are avg. Eight-bit pixel intensity values for each image region.

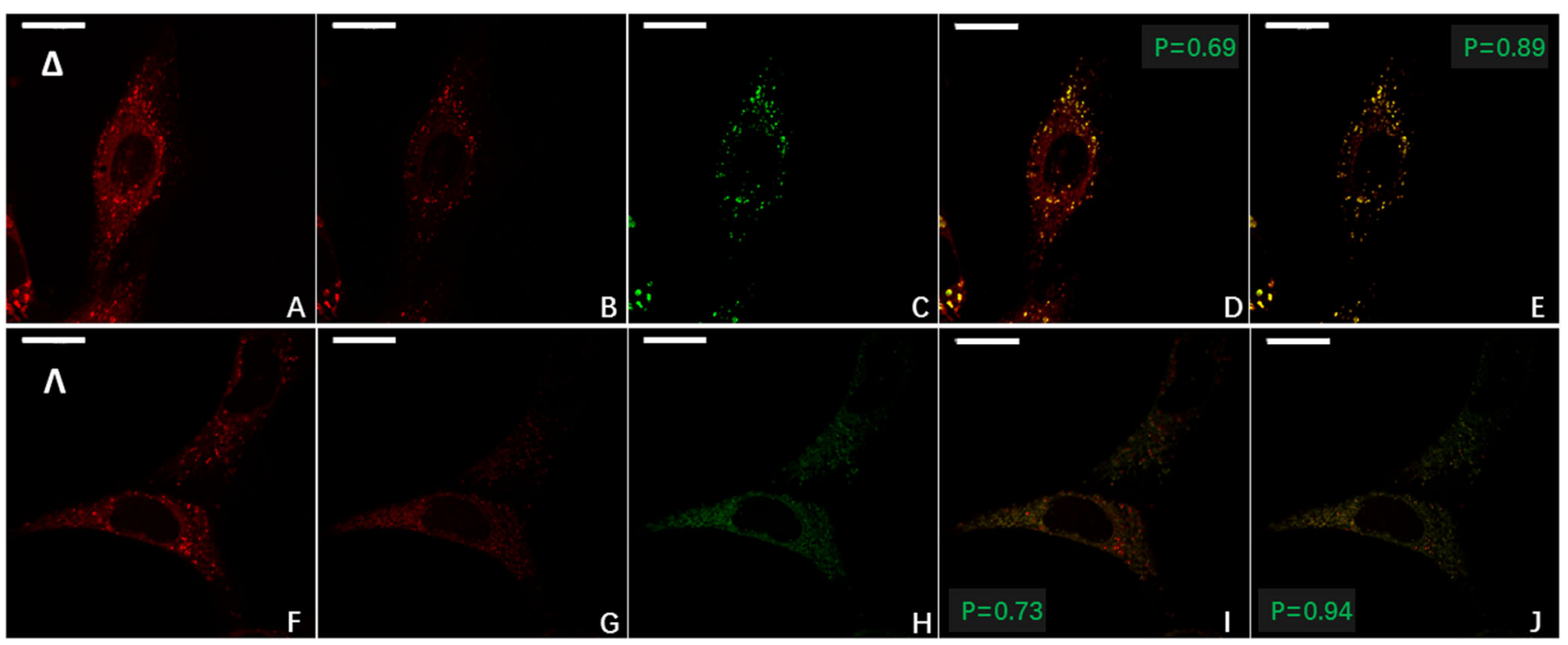

Fig. 6 Enantioselective co-localisation of Eu:L1 with achiral commercial co-stains in live NIH 3T3 cells. Live cell enantioselective differential chiral contrast (EDCC) co-localisation CPL-LSCM of Eu:L1 with commercial co-stains ( $\lambda_{\mathrm{ex}}=488 \mathrm{~nm}, 2 \mathrm{~mW}, \lambda_{\mathrm{em}}=500$ to $\left.530 \mathrm{~nm}\right)$ in NIH $3 T 3(\times 631.4 \mathrm{NA}$ oil objective, $96 \times 96 \mu \mathrm{m}$ FOV, 100 avg., $790 \mathrm{~nm}$ axial section). Top row (A-E) $\Delta$-Eu:L1. A Total europium emission $\left(\lambda_{\mathrm{ex}}=355 \mathrm{~nm}, 20 \mathrm{~mW}, \lambda_{\mathrm{em}}=589\right.$ to $720 \mathrm{~nm}$ ). B Right-handed EDCC image (right CPL-left CPL) highlighting predominantly Lysosomal localisation. C LysoTrackerTM Green. D RGB merge of (A) and (C). E RGB merge of B and C showing enhancement of Pearson's coefficient. Bottom row (F-J) $\Lambda$-Eu:L1. F as per (A). G Left-handed EDCC image (left $\mathrm{CPL}$-right $\mathrm{CPL}$ ) highlighting predominantly mitochondrial localisation. $\mathbf{H}$ MitoTracker ${ }^{\mathrm{TM}}$ Green. I RGB merge of $\mathbf{F}$ and $\mathbf{H}$. J RGB merge of $\mathbf{G}$ and $\mathbf{H}$ showing enhancement of Pearson's coefficient. Scale bars $=20 \mu \mathrm{m}$, green numbers indicate Pearson's coefficient for each RGB merge.

To investigate further the enantioselective localisation of $\Lambda$ and $\Delta$-Eu:L1, we repeated this experiment with the addition of LysoTracker ${ }^{\mathrm{TM}}$ DND-26 Green and MitoTracker ${ }^{\mathrm{TM}}$ FM Green dyes. From the differential CPL-LSCM images (Fig. 6), it can be seen that the $\Delta$-enantiomer strongly overlaps with the emission from LysoTracker ${ }^{\mathrm{TM}}$ DND-26 Green (Pearson's-coefficient $=$ 0.89 , Fig. $6 \mathrm{E}$ ), whereas the $\Lambda$-enantiomer strongly overlaps with that of MitoTracker ${ }^{\mathrm{TM}}$ FM Green (Pearson's-coefficient $=0.94$, Fig. 6J). Without enantioselective differential CPL imaging, these coefficients are significantly reduced (0.69 and 0.73 , Fig. $6 \mathrm{D}, \mathrm{I}$, respectively).

This study unequivocally demonstrates and confirms that the different enantiomers Eu:L1 selectively target different cellular organelles. Enantioselective imaging is a significant milestone in live-cell optical microscopy. The above detailed simple modifications to any existing LSCM enable researchers worldwide to exploit the importance and versatility of chiral bioimaging. This could trigger a paradigm shift in the fields of molecular cell 
biology and chemistry, opening new avenues in bioprobe research by enantioselectively targeting and tracking chiral species.

\section{Discussion}

To conclude, we have presented three major advances in the intertwined disciplines of CPL spectroscopy and chiral luminescence imaging. Firstly, we have demonstrated the CPL-LSCM system, which is capable of rapidly and simultaneously acquiring diffraction-limited enantioselective chiral-contrast based CPLdifferential images for the sub-cellular tracking of emissive chiral sub-cellular probes. This milestone is accompanied by the proofof-concept demonstration of 2PE-CPL spectroscopy, showing that low-energy 2PE-CPL-LSCM is ripe for future development. Secondly, we have created a well-controlled inert CPL test target for use as a standard for enantioselective chiral-contrast imaging. This test target consists of enantiopure solutions of a suitable europium complex simultaneously drop-cast in a spatially separated manner directly onto conventional glass microscope slides. This simple test target can serve to compare and benchmark future enantioselective chiral-contrast CPL differential imaging systems that may be developed by the multi-disciplinary imaging community. Thirdly, we have imaged $\Lambda$ - and $\Delta$-enantiomers of a known chiral europium complex that localises to different organelles within mouse skin fibroblast (NIH 3T3) cells, with each enantiomer showing an excellent co-localisation profile with common lysosomal and mitochondrial tracker dyes.

We anticipate that these developments will open uncharted research avenues for fundamental studies of chiral interactions in the domains of chemistry and molecular biology. CPL-LSCM will be a vital new tool in optical microscopy aiding the development of chiral bio-probes to be taken to the next level. In material sciences, CPL-LSCM could be used for characterising 3D display technologies (e.g. emissive chiral polymers) and verification of physically unclonable stochastically micro-patterned CPL-active security inks ${ }^{16-18}$. Ultimately, the work presented here opens a new window into the world of chiral molecular interactions which is ripe for exploration by shedding light on the previously unexploited chiral biochemical processes that fundamentally underpin life.

\section{Methods}

EDCC imaging. Microscope control and image acquisition and analysis were performed with Leica's commercial microscope software LAS-X. EDCC imaging is achieved using Image J software (v1.49) ${ }^{31}$, with its built-in image calculator add-on software by subtracting one CPL channel from the other, and vice versa. The convention used herein is: left-handed enantioselective contrast $=$ left $\mathrm{CPL}$-right CPL. Right-handed enantioselective contrast $=$ right CPL-left CPL. Images for a typical $1024 \times 1024$ pixel FOV with 10 line accumulation bidirectional scanning sequence were typically acquired in $90 \mathrm{~s}$ (corresponding to 10 full-frame accumulations). Confocality-axial ( $\mathrm{z}$ ) resolution-was governed by the applied pinhole diameter denoted in Airy disk units to preserve resolution and maximise light detection from the FOV.

Images were obtained using the detector saturation mode of LAS-X where each image is assessed for maximum intensity value. Each image is only recorded if no $4 \times 4$ pixel cluster (Nyquist sampling covering an area determined by the systems optical resolution, $126 \times 126 \mathrm{~nm}$ at $355 \mathrm{~nm}$ excitation using $1.4 \mathrm{NA}$ objective) possesses average intensity values of 255 on an 8 -bit greyscale. This allows the employed gain of each detector to be synchronised and kept constant, so no error associated with pixel intensity saturation is included accidentally contributing to pixel uncertainties, lowering $\mathrm{S} / \mathrm{N}$ and exponentially increasing the limit of detection values.

Non-live cell 8-bit average pixel chiroptical contrast value calculations were facilitated by selecting and averaging five different positions non-overlapping equal size and shape arbitrary area of the sample with respect to each enantiomer and dark background. Due to the $1024 \times 1024$ pixel size of each recorded image total FOV, this arbitrary area has been kept at a constant area of $100 \times 100$ pixels region of interest (ROI). The average maximum 8-bit grayscale pixel intensity values were determined using the LSCM's built-in LAS-X software that is employing a maximum average value ROI histogram methodology that is based on standard Gaussian distribution profiling of the average intensity values. Due to the employed methodology and the averaging nature of image acquisition and ROI calculation the limit of detection (error associated with) 8-bit greyscale contrast value is below $1 \%$ ( $<3$ average greyscale value on a $0-255$ pixel intensity scale).

During live-cell imaging, adaptations of the above detailed chiroptical contrast greyscale value determination methodology were applied. In this case, full FOV 8-bit contrast values have been calculated using the LAS-X software. To eliminate errors associated with the number of cells occupying the FOV-in other words, the variable amount of dark 'black' background in each image, this value has been corrected with a below limit of detection value correction. In each case, the software only uses pixel for the average 8-bit grayscale intensity value determines if the intensity value of the pixel is above the value of 4 (on a $0-255$ pixel intensity scale). This is determined using the total Europium emission image and the selected arbitrary ROI area selection is then kept identical throughout the imaging sequence resulting in high precision chiroptical contrast calculations.

Pearson correlation coefficients were calculated following standardised protocols using the corresponding channel of the colour split raw images using the ImageJ-JACoP plug in ${ }^{33}$.

Statistics and reproducibility. Where instruments incorporating a scanning monochromator have been used (absorption, emission, and excitation spectra) each sample have been recorded and averaged as triplicate measurements. Spectra, where CCD detectors have been employed, such as two-photon cross-section determination, multiphoton and CPL spectroscopy, have been measured as an average of a thousand spectra on triplicate samples.

Microscopy images presented herein are representative images of the experiments discussed. Each experiment has been repeated in triplicates and each sample has been recorded and studied recording a minimum of five separate imaging sequences.

Imaging parameters presented, such as brightness and chiroptical contrast have been calculated on each individual imaging sequence according to the protocol detailed in the Methods and the Supplementary Information (SI) section.

Reporting summary. Further information on research design is available in the Nature Research Reporting Summary linked to this article.

\section{Data availability}

All data generated and analysed, including spectra and raw microscopy images, during this study are available from the corresponding author upon request.

\section{Code availability}

Custom codes are written in Labview2013 and Matlab2019b developed and used during this study are available from the corresponding author upon request.

Received: 9 June 2021; Accepted: 15 December 2021; Published online: 27 January 2022

\section{References}

1. Blackmond, D. G. The origin of biological homochirality. Cold Spring Harb. Perspect. Biol. 2, a002147-a002147 (2010).

2. Tokunaga, E., Yamamoto, T., Ito, E. \& Shibata, N. Understanding the thalidomide chirality in biological processes by the self-disproportionation of enantiomers. Sci. Rep. 8, 6-12 (2018).

3. Singh, J. \& Hagen, T. J. Chirality and Biological Activity. in Burger's Medicinal Chemistry and Drug Discovery (John Wiley \& Sons, Inc., 2010). https:// doi.org/10.1002/0471266949.bmc016.pub2.

4. Yamada, T. et al. Synthesis of chiral Labtb and visualization of its enantiomeric excess by induced circular dichroism imaging. Chemistry 25, 6698-6702 (2019)

5. Zhou, Y., Li, H., Zhu, T., Gao, T. \& Yan, P. A highly luminescent chiral tetrahedral Eu4L4(L')4 cage: chirality induction, chirality memory, and circularly polarized luminescence. J. Am. Chem. Soc. 141, 19634-19643 (2019)

6. Mawatari, K., Kubota, S. \& Kitamori, T. Sensitive chiral analysis method for microchip by circular dichroism UV thermal lens microscope (UV-CD-TLM). Micro Total Anal. Syst. 78, 1582-1584 (2006).

7. Narushima, T. \& Okamoto, H. Circular dichroism microscopy free from commingling linear dichroism via discretely modulated circular polarization. Sci. Rep. 6, 1-10 (2016).

8. Doistau, B., Jiménez, J.-R. \& Piguet, C. Beyond chiral organic (p-block) chromophores for circularly polarized luminescence: the success of d-block and f-block chiral complexes. Front. Chem. 8, 1-27 (2020).

9. Frawley, A. T., Pal, R. \& Parker, D. Very bright, enantiopure europium(III) complexes allow time-gated chiral contrast imaging. Chem. Commun. 52, 13349-13352 (2016)

10. Miller, T. L. et al. Calcium binding sites of rabbit troponin and carp parvalbumin. FEBS Lett. 58, 1-3 (1975). 
11. Brittain, H. G. \& Richardson, F. S. Circularly polarized emission studies on the chiral nuclear magnetic resonance lanthanide shift reagent tris(3trifluoroacetyl-d-camphorato)europium(III). J. Am. Chem. Soc. 1, 5858-5863 (1976).

12. Tsumatori, H., Harada, T., Yuasa, J., Hasegawa, Y. \& Kawai, T. Circularly polarized light from chiral lanthanide(III) complexes in single crystals. Appl. Phys. Express 4, 011601 (2011).

13. Koike, H., Nozaki, K. \& Iwamura, M. Microscopic imaging of chiral amino acids in agar gel through circularly polarized luminescence of Eu III complex. Chemistry 15, 85-90 (2020).

14. Schermelleh, L., Heintzmann, R. \& Leonhardt, H. A guide to super-resolution fluorescence microscopy. J. Cell Biol. 190, 165-175 (2010).

15. MacKenzie, L. E., Pålsson, L.-O., Parker, D., Beeby, A. \& Pal, R. Rapid timeresolved circular polarization luminescence (CPL) emission spectroscopy. Nat. Commun. 11, 1676 (2020).

16. MacKenzie, L. E. \& Pal, R. Circularly polarized lanthanide luminescence for advanced security inks. Nat. Rev. Chem. https://doi.org/10.1038/s41570-02000235-4 (2020).

17. Arppe, R. \& Sørensen, T. J. Physical unclonable functions generated through chemical methods for anti-counterfeiting. Nat. Rev. Chem. 1, 0031 (2017).

18. Wan, L. et al. Inverting the handedness of circularly polarized luminescence from light-emitting polymers using film thickness. ACS Nano 13, 8099-8105 (2019).

19. Lunkley, J. L., Shirotani, D., Yamanari, K., Kaizaki, S. \& Muller, G. Chiroptical spectra of a series of tetrakis $((+)-3$-heptafluorobutylyrylcamphorato) lanthanide(III) with an encapsulated alkali metal ion: circularly polarized luminescence and absolute chiral structures for the $\mathrm{Eu}(\mathrm{III})$ and $\mathrm{Sm}(\mathrm{III})$ complexes. Inorg. Chem. 50, 12724-12732 (2011).

20. Kumar, J., Marydasan, B., Nakashima, T., Kawai, T. \& Yuasa, J. Chiral supramolecular polymerization leading to eye differentiable circular polarization in luminescence. Chem. Commun. 52, 9885-9888 (2016).

21. Arrico, L., Di Bari, L. \& Zinna, F. Quantifying the overall efficiency of circularly polarized emitters. Chemistry 27, 2920-2934 (2021).

22. Denk, W., Strickler, J. H. \& Webb, W. W. Two-photon laser scanning fluorescence microscopy. Science 248, 73-76 (1990).

23. Liu, D. et al. Near-infrared light activates molecular nanomachines to drill into and kill cells. ACS Nano 13, 6813-6823 (2019).

24. Chen, W. et al. Two-photon absorption-based upconverted circularly polarized luminescence generated in chiral perovskite nanocrystals. J. Phys. Chem. Lett. 10, 3290-3295 (2019).

25. Bruce, J. I., Parker, D., Lopinski, S. \& Peacock, R. D. Survey of factors determining the circularly polarised luminescence of macrocyclic lanthanide complexes in solution. Chirality 14, 562-567 (2002).

26. Starck, M., MacKenzie, L. E., Batsanov, A. S., Parker, D. \& Pal, R. Excitation modulation of Eu:BPEPC based complexes as low-energy reference standards for circularly polarised luminescence (CPL). Chem. Commun. 55, 14115-14118 (2019).

27. Xu, C. \& Webb, W. W. Measurement of two-photon excitation cross sections of molecular fluorophores with data from 690 to $1050 \mathrm{~nm}$. J. Opt. Soc. Am. B 13, 481 (1996).

28. Frawley, A. T., Linford, H. V., Starck, M., Pal, R. \& Parker, D. Enantioselective cellular localisation of europium (III) coordination complexes. Chem. Sci. 9, 1042-1049 (2018).

29. Pålsson, L.-O., Pal, R., Murray, B. S., Parker, D. \& Beeby, A. Two-photon absorption and photoluminescence of europium based emissive probes for bioactive systems. Dalt. Trans. https://doi.org/10.1039/b710717j (2007).

30. Makarov, N. S., Drobizhev, M. \& Rebane, A. Two-photon absorption standards in the 550-1600 nm excitation wavelength range. Opt. Express 16, 4029 (2008).

31. Schneider, C. A., Rasband, W. S. \& Eliceiri, K. W. NIH Image to ImageJ: 25 years of image analysis. Nat. Methods 9, 671-675 (2012).

32. Fradgley, J. D., Frawley, A. T., Pal, R. \& Parker, D. Striking solvent dependence of total emission and circularly polarised luminescence in coordinatively saturated chiral europium complexes: solvation significantly perturbs the ligand field. Phys. Chem. Chem. Phys. 23, 11479-11487 (2021).

33. Bolte, S. \& Cordelières, F. P. A guided tour into subcellular colocalization analysis in light microscopy. J. Microsc. 224, 213-232 (2006).

\section{Acknowledgements}

R.P. acknowledges support from the Royal Society University Research Fellowship URF $\mid R \backslash 191002$ and H2020-MSCA-ITN-859752 HEL4CHIROLED. R.P. and P.S. acknowledge support from BBSRC BB/S017615/1, L.M. acknowledges support from the BBSRC Discovery Fellowship BB/T009268/1. P.S. would like to thank Dr. Piotr Pander for his assistance with spin-coating. R.P. thanks Prof. Andrew Beeby for the stimulating discussions regarding CPL spectroscopy. D.P. thanks Dr. Matthieu Starck and Dr. Andrew Frawley for the synthesis of Eu complexes used herein. We are also thankful for the ongoing support of Leica Microsystems UK (Dr. Malcolm Lang, Brian Preston and Jason Lewis) and Coherent UK (Graham Wright and Blair Welsh).

\section{Author contributions}

P.S. ${ }^{1}$ Co-constructed the CPL-LSCM performed all blotting and casting experiments and drafted/edited the paper. L.M.: ${ }^{1}$ Co-constructed the CPL-LSCM, Co-designed SS-CPL and edited the paper. D.P.: Secured funding for the proof-of-concept epifluorescence microscope with R.P., provided lanthanide complexes for analysis and edited the paper. R.P.: Secured project funding, designed and constructed the confocal CPL microscope, performed multi-photon and live-cell experiments, and drafted/edited the paper. ${ }^{1} \mathrm{Co}$-first authors can prioritise their names when adding this paper's reference to their resumes.

\section{Competing interests}

R.P. is an inventor on patent WO2016174395A1: Light detecting apparatus for simultaneously detecting left- and right-handed circularly polarised light. The authors declare no competing interests.

\section{Additional information}

Supplementary information The online version contains supplementary material available at https://doi.org/10.1038/s41467-022-28220-z.

Correspondence and requests for materials should be addressed to Robert Pal.

Peer review information Nature Communications thanks the anonymous reviewers for their contribution to the peer review of this work. Peer reviewer reports are available.

Reprints and permission information is available at http://www.nature.com/reprints

Publisher's note Springer Nature remains neutral with regard to jurisdictional claims in published maps and institutional affiliations.

Open Access This article is licensed under a Creative Common Attribution 4.0 International License, which permits use, sharing, adaptation, distribution and reproduction in any medium or format, as long as you give appropriate credit to the original author(s) and the source, provide a link to the Creative Commons license, and indicate if changes were made. The images or other third party material in this article are included in the article's Creative Commons license, unless indicated otherwise in a credit line to the material. If material is not included in the article's Creative Commons license and your intended use is not permitted by statutory regulation or exceeds the permitted use, you will need to obtain permission directly from the copyright holder. To view a copy of this license, visit http://creativecommons.org/ licenses/by/4.0/

(C) The Author(s) 2022 